Revista de Estudios Histórico-Jurídicos

[Sección Derecho Romano]

XXXII (Valparaíso, Chile, 2010)

[pp. 107 - 118]

\title{
"Propter IURIS CONDICIONEM IN SUSPENSO EST". LA “CONDICIO SUPERVACUA”, LA "CONDICIO EXTRINSECUS" Y LA CONDICIÓN DE DERECHO EN EL DERECHO ROMANO CLÁSICO
}

["Propter iuris condicionem in suspenso est". "condicio supervacua”, "condicio extrinsecus" and the Condition of Law in "Classic Roman Law"]

\author{
Caroline Mokrejs* \\ Universidad de Viena
}

\section{RESUMEN}

El artículo trata de la condicio iuris en el Derecho romano clásico e intenta analizar su definición y estudiar las fuentes más importantes, que son $\mathrm{D} 21,1,43,10$, D. $35,1,21$, D $35,1,99$ y D $36,2,25,1$. La condicio iuris ha sido relacionada e identificada con la condicio extrinsecus y la condicio supervacua, que son dos tipos que carecen de efecto jurídico. Por este mismo motivo se ha considerado que la condicio iuris forma parte de la noción de la condicio tacita, lo cual parece ser correcto si se trata de la definición de la condicio iuris "in abstracto". Pero al analizar los fragmentos claves se pone de manifiesto que este concepto y los textos mismos se contradicen hasta cierto punto. Por lo tanto, no parece ser posible establecer una definición generalizada de la condicio iuris en el Derecho romano clásico.

Palabras clave: condicio iuris - Condicio extrinsecus - Condicio supervacua Condicio tacita.

\begin{abstract}
This article refers to the condicio iuris in classic Roman Law and intends to analyse its definition and study the most important sources, which are D 21,1,43,10, D.35,1,21, D 35,1,99 y D 36,2,25,1. Condicio iuris has been related and identified with condicio extrinsecus and condicio supervacua, two types that lack legal effect. This is why condicio iuris is part of the condicio tacita notion, which seems to be right if it is the definition of "in abstracto" condicio iuris. However, when analysing the key fragments, it is clear that up to a certain point this concept and the texts themselves are contradictory. Therefore, it seems that a general definition of condicio iuris in classic Roman Law is not possible.

KEYWORDS: condicio iuris - Condicio extrinsecus - Condicio supervacua - Condicio tacita.
\end{abstract}

* Profesora del Instituto de Derecho Romano y de Historia Antigua del Derecho de la Facultad de Derecho de la Universidad de Viena. Dirección postal: Institut für Römisches Recht und Antike Rechtsgeschichte, Rechtswissenschaftliche Fakultät, Universität Wien, Schenkenstr. 8-10,1010 Wien, Austria. Correo electrónico: caroline.mokrejs@univie.ac.at 


\section{INTRODUCCIÓN Y ESTADO DE LA CUESTIÓN}

La condición produce un estado de pendencia, es decir, los efectos jurídicos del negocio condicionado están sometidos a la verificación de un acontecimiento futuro e incierto. Normalmente las partes de un negocio suelen introducir la condición expresamente en su compromiso basado en la voluntad de ambas. Es natural que no sea así en los casos de las llamadas "condiciones tácitas", un concepto que incluye tanto la condicio tacita como la condicio iuris. Por un lado la condicio tacita es una condición que, establecida por la voluntad de las partes, se halla sobreentendida en el contenido del negocio, mientras que la condicio iuris ("Rechtsbedingung"1) se define como condición que no proviene de la voluntad de las partes, sino que está impuesta por una disposición del Derecho objetivo.

El número de estudios sobre la condicio iuris en el Derecho privado romano es limitado. Las publicaciones de fondo (para este estudio) son los artículos de Max Kaser (publicados en 1956 y 1976)² y de Légier (año 1966) ${ }^{3}$, así como la monografía recientemente publicada de Santiago Castán Pérez-Gómez con el título "Condicio iuris" y "tacita condicio". Las condiciones implícitas en el derecho privado romano" .

La mayor parte de las fuentes sobre las condiciones tácitas se presenta muy heterogénea, bien porque se la denomina a veces condicio tacita o bien, condicio iuris, o porque dichas condiciones no estén nombradas expresamente sino que sólo se utilice expresiones que indican la presencia de una condición tácita en el contenido del negocio. ${ }^{5}$ De ahí que Castán Pérez-Gómez resuma la condicio tacita y la condicio iuris bajo un concepto general de una "condicio tacita en sentido lato". Como definición de la condicio iuris dice que "para los romanos la condicio iuris era el acontecimiento futuro y objetivamente incierto del que se hacía depender la producción de efectos de un negocio jurídico por su propia naturaleza, su objeto o por una especial disposición del Derecho"6. Acentúa que es necesario que se diferencie esa condición de la "condicio tacita propiamente dicha", la cual

\footnotetext{
${ }^{1}$ Para lo concerniente a la noción moderna de la "Rechtsbedingung", véase: OERTMAnN, Paul, Die Rechtsbedingung (Leipzig, 1924; reimpresión Aalen, Scientia, 1968).

${ }^{2}$ Kaser, Max, "Condicio iuris" und "condicio tacita", en Symbolae Raphaeli Taubenschlag dedicatae (1956), I, pp. 427 ss.; con algunas correcciones después en: KASER, Max, Ausgewählte Schriften (Napoli, Jovene, 1976), II, pp. 225 ss.

${ }^{3}$ LÉGIER, "Tacita condicio", en RHDFE., 44 (1966), pp. 5 ss.

${ }^{4}$ Castán Pérez-Gómez, Santiago, "Condicio iuris" y "tacita condicio". Las condiciones implícitas en el Derecho privado romano (Madrid, Dykinson, 2006).

${ }^{5}$ Véanse: D. 23,3,21 (Ulp., 35 Sab.): "constat habere in se condicionem"; D. 23,3,68 (Papin, 10 quaest.); D. 26,2,10,4 (Ulp., 36 Sab.): "tacitam condicionem accipiat"; D. 35,1,1,3 (Pomp., 3 Q. Mucium): "videtur inesse haec condicio" - "Inest autem condicio".

${ }^{6}$ Castán Pérez-Gómez, S., "Condicio iuris" y "tacita condicio", cit. (n. 4), p. 44. En este sentido, véase: KaSER, M., "Condicio iuris", cit. (n. 2), p. 422: "Die Rechtsbedingung [beruht aber] nicht auf dem erklärten Parteiwillen, der durch die Beifügung der Bedingung die Wirkungen eines Geschäfts einschränkt, sondern unmittelbar auf einem Rechtssatz oder, was dem gleichsteht, auf dem Wesen - dem Inbegriff der Merkmale - des verwendeten Rechtsgeschäfts".
} 
proviene de la voluntad de las partes ${ }^{7}$, así como de los presupuestos de validez de los negocios jurídicos y de las "condiciones necesarias". Esa concepción de la condicio iuris la utiliza, no sólo en la interpretación de las fuentes nombrando expresamente la condicio iuris, sino también la aplica de forma mucho más amplia, como por ejemplo en el ámbito de la dote o en las disposiciones testamentarias. Analizar y verificar dicha categorización es objeto de este estudio.

\section{LAS FUENTES QUE SE REFIEREN EXPRESAMENTE A LA “CONDICIO IURIS”}

En las fuentes se encuentran sólo dos textos que nombran expresamente la condicio iuris. Sobre todo el siguiente fragmento es el que plantea algunas preguntas sobre la naturaleza de la condicio iuris y se encuentra en D. 35,1,21 (Iul., 31 dig.): "Multum interest, condicio facti an iuris esset: nam huiusmodi condiciones 'si navis ex Asia venerit' 'si Titius consul factus erit', quamvis impletae essent, impedient heredem circa adeundam hereditatem, quamdiu ignoraret eas impletas esse: quae vero ex iure venient, in his nihil amplius exigendum, quam ut impletae sint. veluti si quis se filium familias existimat, cum sit pater familias, poterit adquirere hereditatem: quare et ex parte heres scriptus, qui ignorat, an tabulae testamenti apertae sint, adire hereditatem poterit".

En sus estudios sobre la condicio iuris del año 1956, Kaser considera la antítesis entre la condicio facti y la condicio iuris como equivocada y por lo tanto da el texto entero como posclásico ${ }^{8}$. En D. 35,1,21, Juliano distingue entre la condicio facti, para la cual pone el ejemplo "si navis ex Asia venerit", y la condicio iuris, que "ex iure venie $[\mathrm{n}] t$ ", es decir que proviene de una disposición del Derecho objetivo. El jurista diferencia entre estas dos formas de condiciones a causa de que, dependiendo de cuál se trate, los efectos jurídicos concernientes a la adquisición de la herencia varían. En el caso de la condicio iuris, o sea, el paterfamilias que se cree aún filius familias, el jurista exige solamente la verificación de la "condición de Derecho", mientras que la condicio facti no sólo necesita verificarse, sino también el conocimiento de este hecho por parte del heredero.

Puesto que la condición de Derecho tiene como objeto la verificación de un acontecimiento futuro e incierto, produce -igualmente que la condicio facti- un estado de pendencia. No obstante, este estado no está impuesto por la voluntad de las partes, sino proviene del Derecho objetivo", o sea, una especial disposición del Derecho. En el caso del putativo filius familias, está claro que ser sui iuris es un requisito de validez para la adquisición de la herencia. Sin embargo dicho acontecimiento de ser sui iuris carece de incertidumbre porque ya se ha cumplido. No hay estado de pendencia en esta situación porque es cierto que el heredero es

${ }^{7}$ Véase: Castán Pérez-Gómez, "Condicio iuris" y "tacita condicio“, cit. (n. 4), pp. 31 ss.

${ }^{8}$ Véase: Kaser, M., “Condicio iuris”, cit. (n. 2), p. 423. Este autor corrigió su posición en 1976 y se mostró convencido de la clasicidad de este fragmento; así: KASER, Ausgewählte Schriften, cit. (n. 2), II, p. 242. Aunque sea la única distinción transmitida entre "condicio iuris" y "condicio tacita", Castán Pérez-Gómez, S., "Condicio iuris" y "tacita condicio", cit. (n. 4), p. 39, considera el fragmento clásico y no interpolado, porque se podría pensar que esta distinción fuera una creación del jurista Juliano. 
paterfamilias en el momento de la adquisición, ya que en el texto se pone claramente de manifiesto que la verificación de dicho acontecimiento ha tenido lugar?

Como el concepto de la condicio iuris no coincide con el de la condicio iuris del fragmento D. 31,1,21, hay que analizar el segundo texto que habla expresamente de la condición de Derecho y se encuentra en D. 21,1,43,10 (Paul., 1 ed. aedilium curulium): "Interdum etiamsi pura sit venditio, propter iuris condicionem in suspenso est, veluti si servus, in quo alterius usus fructus, alterius proprietas est, aliquid emerit: nam dum incertum est, ex cuius re pretium solvat, pendet, cui sit adquisitum, et ideo neutri eorum redhibitoria competit".

En el usufructo del esclavo todas las adquisiciones pertenecen al usufructuario, que son hechas por el esclavo con medios propios del titular del usufructo (" $e x$ re") o con su propio trabajo ("ex operis suis"). Todas las adquisiciones que se pagan de manera diferente pertenecen al propietario del esclavo. ${ }^{10}$ En D. 21,1,43,10, el esclavo ya ha concluido el contrato de compraventa, aunque todavía no se haya pagado el precio. No está claro, si el esclavo pagará el precio ex operis suis, ex re del usufructuario o con otros medios. Por lo tanto no está claro quién se hará propietario de la adquisición, el dueño del esclavo o el usufructuario, y a continuación, a quién le corresponderá la actio redhibitoria.

Orientándose en las "exigencias" de una condicio iuris, este caso tiene bastante en común con la condición de Derecho. El objeto principal de la condición no tiene su origen en la voluntad de las partes, sino que el estado de pendencia proviene de las disposiciones del Derecho objetivo concernientes a la adquisición de la posesión. Por lo tanto, la condición surge de una disposición del Derecho objetivo lo que permite calificarla como proveniente "ex iure"11.

\section{III. ¿OTROS Campos de aplicación DE LA “CONDicio IURIS”?}

Según algunos autores ${ }^{12}$, la condicio iuris no está limitada a estos dos textos, sino que ha sido aplicada por los juristas romanos en otros ámbitos. Castán

${ }^{9}$ Kaser, M., Ausgewählte Schriften, cit. (n. 2), p. 243: "Auf seine Kenntnis seines wahren Status kommt es nicht an. Solange er diese Kenntnis nicht hat, besteht freilich, anders als in Paul. D.21.1.43.10, nur ein scheinbarer, kein wirklicher Schwebezustand." Por mi parte no hay un pseudo estado de pendencia, porque el heredero está convencido de ser filius familias y por eso simplemente no existe estado de incertidumbre, ni siquiera por parte subjetiva del heredero.

${ }^{10}$ Véase, Kaser, RPR., I, p. 263.

${ }^{11}$ Por mi parte, la calificación de esta compraventa como condicional no me parece apropiada. El contrato mismo es válido y puro, aunque ciertos efectos jurídicos, como por ejemplo la actio rehibitoria, estén sometidos a dicha condicio iuris. Véase: CASTÁn Pérez-Gómez, "Condicio iuris" y "tacita condicio", cit. (n. 4), p. 145: "El jurista Paulo quiere establecer una diferencia entre una compraventa pura y otra sometida a condicio iuris [...]".

${ }^{12}$ En la stipulatio dotis causa, WACKE, Andreas, Zur causa der stipulation, en TR. 40 (1972), p. 248, considera la celebración del matrimonio como condicio iuris; KASER, en Symbolae Raphaeli Taubenschlag dedicatae, cit. (n. 2), p. 430, también extiende el campo de aplicación de la condicio iuris al identificarla como "(condicio) extrinsecus exspectanda", la que él contrapone a la "condicio ex testamento" (sea como antítesis a la condicio tacita); véase: D. 36,2,25,1 (Papin., 18 quaest.). 
Pérez-Gómez interpreta como condicio iuris lo que en otros textos, como los de D. $28,5,70 ; 23,3,21 ; 36,2,25 ; 36,2,22,1$ y $35,1,99^{13}$, se denomina condición implícita.

\section{Fuentes sobre la "dos".}

Aplicando su sistema de condiciones al caso de la dote, Castán Pérez-Gómez verifica ${ }^{14}$ que los textos sobre la dote ${ }^{15}$ no se refieren a la condicio tacita, sino más bien a la condicio iuris ${ }^{16}$. El fragmento conservado en D. 23,3,3 $3^{17}$ dice: "neque enim dos sine matrimonio esse potest" y, por lo tanto, la subsiguiente celebración del matrimonio es la condición para la estipulación de la dote ${ }^{18}$. Para Castán Pérez-Gómez aquella condición no es una "condicio tacita propiamente dicha" porque no proviene de la voluntad de las partes sino del Derecho objetivo. Según mi opinión no es apropiado limitarse estrictamente al concepto de la condicio iuris en el caso presente, ya que, si bien es cierto que la condicio iuris tiene su origen en una disposición del Derecho, es probable que ésta provenga de una aplicación frecuente en las estipulaciones de la dote. ${ }^{19}$ Teniendo en cuenta que las fuentes no nombran ni una sola vez la condicio iuris, la opinión de Kaser me parece más apropiada.

Leemos en D. 23,3,21 (Ulp., 35 Sab.) 20: "Stipulationem, quae propter causam dotis fiat, constat habere in se condicionem hanc 'si nuptiae fuerint secutae', et ita demum ex ea agi posse (quamvis non sit expressa condicio), si nuptiae, constat: quare si nuntius remittatur, defecisse condicio stipulationis videtur".

La condición implícita expresada en la frase: "habere in se condicionem" consiste en la formulación "si nuptiae fuerint secutae" 21 , es decir, la subsiguiente celebración del matrimonio es la condición para la validez de la estipulación de la dote ${ }^{22}$.

${ }^{13}$ Véase: Castán Pérez-Gómez, "Condicio iuris" y "tacita condicio", cit. (n. 4), p. 36.

${ }^{14}$ En otro sentido véase: KaSER, Symbolae Taubenschlag, cit. (n. 2), p. 433: "Die c.t. der künftigen Eheschliessung ist im Fall der vor der Ehe abgeschlossenen Dotalstipulation eine 'echte' Bedingung, keine blosse Rechtsbedingung. Denn sie enthält eine durch den Parteiwillen dem Stipulationsgeschäft beigesetzte, wenn auch äusserlich hinter den Worten 'dotis causa' verdeckte Einschränkung durch ein Schwebeverhältnis, das erst durch Eintritt eines zukünftigen ungewissen Ereignisses beendet werden soll”.

${ }^{15}$ Véase: Castán Pérez-Gómez, "Condicio iuris" y "tacita condicio", cit. (n. 4), pp. 118 s; KASER, RPR., I, pp. 332 ss; también KÖNIG, Die vor der Ehe bestellte dos nach klassischem römischen Recht, en SDHI., 29 (1963), pp. 152 ss.

${ }^{16}$ Véase Castán Pérez-Gómez, "Condicio iuris" y "tacita condicio", cit. (n. 4), p. 125.

${ }^{17}$ D. 23,3,3 (Ulp., 63 ed.): "Dotis appellatio non refertur ad ea matrimonia, quae consistere non possunt: neque enim dos sine matrimonio esse potest. ubicumque igitur matrimonii nomen non est, nec dos est".

${ }^{18}$ En otro sentido: LÉGIER, cit. (n. 3), p. 26: "Le droit classique ignore donc la condition tacite intégrée à une stipulation de dot, comme il rejette toute condicion tacite appliquée à la stipulatio“.

${ }^{19}$ WACKE, cit. (n. 12), p. 251: "Außerordentliche Häufigkeit".

${ }^{20}$ Castán Pérez-Gómez, "Condicio iuris" y "tacita condicio", cit. (n. 4), p. 125, considera este fragmento como evidentemente interpolado; en otro sentido: KASER, Symbolae Taubenschlag, cit. (n. 2), p. 431; Wolf, Causa stipulationis (Köln - Wien, 1970 ), p. 114 s.

${ }^{21}$ Véase: KASER, RPR., I, p. 336 n. 41.

${ }^{22}$ Véase: Wolf, Causa stipulationis, cit. (n. 20), pp. 90 ss; WACKE, cit. (n. 12), p. 245; 
Otro fragmento de interés en este contexto, en el cual se ha sospechado que existen frases interpoladas ${ }^{23}$ es D. 23.3.68 (Papin., 10 quaest.): "Dotis promissio non ideo minus valebit, quod ignorante initio patre nuptiae non fuerint, si postea consenserit, cum omnis dotis promissio futuri matrimonii tacitam condicionem accipiat. nam et si minor annis duodecim ut maior deducta sit, tunc primum petetur, cum maior annis apud eundem esse coeperit: quod enim volgatum est dotis promissionem in primis dumtaxat nuptiis destinare neque durare obligationem, si post alterius matrimonium ei nubat cui dotem promiserat, tunc locum habet, cum intercesserunt aliae nuptiae".

La primera parte del texto ${ }^{24}$ trata de la estipulación de la dote para un futuro matrimonio, sin el conocimiento del paterfamilias. Si éste da su consentimiento con posterioridad, el matrimonio tendrá validez y la estipulación de la dote produce efectos. Papiniano compara esta situación con el matrimonio de menores de edad. Si una joven que se casó con menos de doce años permanece con su marido hasta cumplir esa edad, el defecto desaparece, el matrimonio es válido y la dote estipulada se puede recaudar ${ }^{25}$.

König decide en favor de la condicio tacita, porque se consigue el contenido de la condición por medio de la interpretación del texto de la stipulatio dotis causa. Por el contrario Wacke habla de una condicio iuris, ya que la celebración del matrimonio es una condición de Derecho para la estipulación ${ }^{26}$. Considero que ambos tienen parte de razón en cierto modo, dado que la condición está expresada en una disposición del Derecho objetivo pero también es probable que tenga sus orígenes en un uso frecuente en la práctica.

Otros fragmentos de interés en el ámbito de la dote "condicionada" son D.

KÖNIG, cit. (n. 15), pp. 174 ss. La stipulatio es nulla, en cuanto es cierto que no se va a celebrar el matrimonio. Véase D. 2,14,4,2 (Paul., 3 ed.): "Huius rei argumentum etiam stipulatio dotis causa facta est: nam ante nuptias male petitur, quasi si hoc expressum fuisset, et nuptiis non secutis ipso iure evanescit stipulatio. idem Iuliano placet”. Como sospechoso de interpolación del fragmento D. 2,14,4,2, véase: Wolf, Causa stipulationis, cit. (n. 20), p. 109. Ya no se puede demandar la estipulación, si el compromiso matrimonial es anulado o si la novia se casa con otro hombre. Véase también: D. 23,3,68; y Wolf, Causa stipulationis, cit. (n. 20), p. 124. Si no obstante se celebra el matrimonio, la estipulación de la dote ya no produce efectos: KÖNIG, cit. (n. 15), p. 193.

${ }^{23}$ Véase: Riccobono, Salvatore, Stipulatio ed instrumentum nel Diritto giustinianeo, en ZSS., rom. Abt., 43 (1922), pp. 262 ss. (pp. 352 ss.); Wolf, Causa stipulationis, cit. (n. 20), p. 120.

${ }^{24}$ Según Wolf, Causa stipulationis, cit. (n. 20), p. 124, el texto está interpolado; pero WACKE, cit. (n. 12), p. 248, disipa el malentendido: "Cum omnis dotis promissio futuri matrimonii tacitam condicionem accipiat" heißt es nun, daß das Mitgiftsversprechen stillschweigend, also nach dem hypothetischen Parteiwillen als durch das Zustandekommen einer gültigen Ehe bedingt anzusehen und damit wirksam ist, obschon der Besteller zuächst glaubte, für eine bereits bestehende Ehe zu promittieren. Dieser Wille ist ein hypothetischer, weil der Promittent -hätte er den Mangel gekannt- ohne Zweifel eine entsprechende Bedingung verabredet hätte".

${ }^{25}$ KÖNIG, cit. (n. 15), pp. 219 s., considera la comparación como no apropiada y por eso sospecha que el fragmento esté interpolado.

${ }^{26}$ Véase WACKE, cit. (n. 12), p. 248. 
23,3,41,127 y D 2,14,4,2 28 , ambos contienen la condición "si nuptiae fuerint secutae“.

\section{Fuentes sobre la "condicio supervacua".}

Según Castán Pérez-Gómez, la aditio hereditatis es otro campo de aplicación de la condicio iuris ${ }^{29}$. Los heredes extranei necesitan un acto de aceptación, la aditio hereditatis, para adquirir la herencia, a diferencia de los heredes necessarii, que adquieren la herencia en el mismo momento de la apertura. En el caso de los heredes extranei, todo los efectos jurídicos del testamento dependen de la aceptación de la herencia sin la cual las disposiciones testamentarias no entrarán en vigor. Hasta ése punto el estado de pendencia en el que se encuentran los efectos jurídicos del testamento se produce por la incertidumbre de la cuestión si el heredero aceptará la herencia o no. Por lo tanto, Castán Pérez-Gómez considera la aceptación de la herencia por el heredero como condicio iuris de la efectividad del testamento ${ }^{30}$. Según él, en este caso se dan todo los requisitos de la condicio iuris, la incertidumbre, así como el origen en una disposición del Derecho objetivo. A mi modo de ver, la calificación como condicio iuris no se presenta tan clara en este caso, ya que -como en el caso de la dote- no se nombra expresamente la condición de Derecho.

Otros fragmentos pertenecientes al ámbito de la aditio hereditatis han sido nombrados por los autores que estudian la condicio iuris.

Así D. 35,1,107 (Gai., l. sing. de casibus): "Aliquando accidit, ut sub condicione datum legatum purum intellegatur, veluti quod sub eadem condicione relictum est, sub qua etiam heres alius institutus est, item quod sub hac condicione relictum est'si hereditatem adierit'. ex diverso quoque purum datum legatum condicionale videtur, veluti quod sub condicione ademptum est, quia sub contraria condicione datum intellegitur".

También D. 36,2,22,1 (Pomp., 5 ad Q. Mucium): "Quaedam autem condiciones etiam supervacuae sunt, veluti si ita scribat: 'Titius heres esto. si Titius hereditatem meam adierit, Maevio decem dato': nam pro non scripto ea condicio erit, ut omnimodo ad heredem Maevii legatum transeat, etiamsi Maevius ante aditam hereditatem decesserit. et idem, si ita fuerit scriptum: 'si Titius hereditatem meam adierit, intra dies centum Maevio decem dato': nam hoc legatum in diem erit, non sub condicione,

${ }^{27}$ D. 23,3,41,1 (Paul., 35 ed.): “Sed si nuptiae secutae non fuerint, ex stipulatu agi non potest: magis enim res quam verba intuenda sunt”. KASER, Symbolae Taubenschlag, cit. (n. 2), p. 433, considera la última parte del fragmento como interpolado, véase.

${ }^{28}$ D. 2,14,4,2 (Paul., 3 ed.): "Huius rei argumentum etiam stipulatio dotis causa facta est: nam ante nuptias male petitur, quasi si hoc expressum fuisset, et nuptiis non secutis ipso iure evanescit stipulatio. idem Iuliano placet”. El fragmento está interpolado según KaSER, Symbolae Taubenschlag, cit. (n. 2), p. 433; también lo considera así WOLF, Causa stipulationis, cit. (n. 20), p. 109.

${ }^{29}$ Véase: Castán Pérez-Gómez, "Condicio iuris" y "tacita condicio", cit. (n. 4), p. 83; KASER, RPR., I, pp. 715 ss.

${ }^{30}$ Véase CAStán Pérez-Gómez, "Condicio iuris" y "tacita condicio", cit. (n. 4), p. 85; en otro sentido LÉGIER, cit. (n. 3), p. 20, que cita el fragmento D. 28,7,12, constatando una "condition nécessaire". 
quia definitio Labeonis probanda est dicentis id demum legatum ad heredem legatarii transire, quod certum sit debitum iri, si adeatur hereditas".

Según Castán Pérez-Gómez, no hay duda de que la aditio hereditatis es una condicio iuris. Ésa conclusión la deduce del hecho de que existe un estado de incertidumbre, si el heredero aceptará la herencia, y porque tiene su origen en el Derecho objetivo. De ahí que todos los efectos jurídicos del testamento dependan de ésa condición.

En el fragmento de D. 35,1,107 se dice, que una condición "si hereditatem adierit" se considera como no existente, es decir, el legado bajo esta "condición" se trata como uno puro. También en D. 36,2,22,1 $1^{31}$ Pomponio verifica, que la condición del legado "si Titius hereditatem meam adierit" se da por superflua, i.e. "supervacua"32. Según Castán Pérez-Gómez, se tendría esta condición "por no puesta” porque ya se encuentra incluida implícitamente y por no ser redundante ${ }^{33}$. En cambio se negaba los efectos de esta "condición", sea implícita o expresa, ya que la imposición de una condición tendría efectos negativos para el legatario y sus herederos. El dies cedens es el momento en el que la expectativa de derecho al legado se convierte en heredable. Ese momento coincide normalmente con el momento del fallecimiento del testador ${ }^{34}$. De morirse el legatario antes del dies cedens no puede transmitir la expectativa de derecho al legado a sus herederos. ${ }^{35} \mathrm{Si}$ se introduce una condicio iuris, es decir, si se hace depender el legado de la aditio hereditatis, el dies cedens no será el día del fallecimiento del testador sino el momento de la aceptación de la herencia. En caso de que el legatario muriera antes de la aditio hereditatis su expectativa de derecho al legado no será heredable. De ahí que sea de interés del legatario, y probablemente también del testador, no retardar el momento del dies cedens. Se concluye de esto que, aun cuando se considere que se trata de una condicio iuris, ésta no produce ningún efecto. No obstante, no se menciona la expresión "condicio iuris" ni una sola vez en todo el texto, todo lo que se puede decir al respecto es que los elementos de la condicio iuris coinciden en este caso.

\section{Fuentes sobre la "condicio extrinsecus".}

En los estudios sobre la condicio iuris no sólo se identifica ésta con la "condicio supervacua" sino también con la llamada "condicio extrinsecus". Esta comparación

${ }^{31}$ Masi, Antonio, Studi sulla condizone nel Diritto romano (Milano, Giuffrè, 1966), p. 19 n. 22.

${ }^{32}$ Kaser, Symbolae Taubenschlag, cit. (n. 2), p. 426: "Die c.i. einem Rechtsgeschäft beizufügen, wäre allerdings wirkungslos, weil sie dort überflüssig ist. So etwa, wenn ein Legat an die Bedingung gebunden wird, dass der Erbe die Erbschaft antritt; hier gilt die Bedingung als nicht geschrieben"..

${ }^{33}$ Véase: Castán Pérez-Gómez, "Condicio iuris" y “tacita condicio", cit. (n. 4), p. 41.

${ }^{34}$ Véase: D. 36,2,5,1 (Ulp., 20 Sab.): "Itaque si purum legatum sit, ex die mortis dies eius cedit: si vero post diem sint legata relicta, simili modo atque in puris dies cedit, nisi forte id fuit legatum, quod ad heredem non transit: nam huius dies non ante cedit, ut puta si usus fructus sit post annum relictus: hanc enim sententiam probamus".

${ }^{35}$ Véase, e contrario, D. 36,2,5 pr (Ulp., 20 Sab.): "Si post diem legati cedentem legatarius decesserit, ad heredem suum transfert legatum". 
se explica con el argumento de que "extrinsecus" significa "desde fuera" y que también la condicio iuris proviene "de fuera", porque no tiene su origen en la voluntad de las partes. Los siguientes fragmentos nos muestran que la condicio extrinsecus, como la condicio supervacua, no produce efectos.

D. 36,2,25,1 (Papin., 18 quaest.): “'Heres meus Titio dato quod mihi Seius debet'. si Seius pupillus sine tutoris auctoritate nummos accepit nec locupletior factus est et creditor ad praesens debitum verba rettulit, quia nibil Seius debet, nullius momenti legatum erit: quod si verbo debiti naturalem obligationem et futuram solutionem cogitavit, interim nihil Titius petet, quasi tacite condicio inserta sit, non secus ac si ita dixisset: 'Titio dato, quod pupillus solverit' vel si legasset 'quod ex Arethusa natum erit' vel 'fructus, qui in illo fundo nascentur'. Contrarium non est, quod, si medio tempore legatarius moriatur et postea partus edatur, fructus perveniant, pecuniam pupillus exsolvat, heres legatarii petitionem habet: namque dies legati, cui condicio non adscribitur, quamvis extrinsecus exspectanda sit, cedit".

El fragmento de D. 36,2,25,1 trata varias cuestiones bastante complejas. La primera parte del texto trata de la "deuda" de un pupillus que (careciendo de ejecutabilidad) se interpreta por la voluntad del testador. Ahora el legado se considera dependiente de una condición implícita, a saber, que se pague la deuda. Este caso también se compara con ejemplos de objetos de un legado, que todavía no existen, como por ejemplo el hijo de la Arethusa que va a nacer o el tal fundo que va a producir frutos. Papiniano concluye que semejantes condiciones no influyen en el dies cedens y que hay que tratar el legado como puro, igualmente que en los casos de la condicio supervacua o extrinsecus. Mejor dicho, se trata del caso en el que el legatario se muere con posterioridad al día del fallecimiento del testador, pero antes del momento de la verificación de la condicio iuris, es decir de la aditio hereditatis. No obstante, el jurista decidió en este caso, que la expectativa de adquerir el legado se transmitía al heredero del legatario. La última frase del fragmento indica, que la condicio extrinsecus -la condición que viene "desde fuera"- no tiene efectos, es decir se tiene el legado como puro. También el fragmento siguiente en el orden palingenético, D. 35,1,99 ${ }^{36}$, trata de la condicio extrinsecus que no viene del testamento mismo sino "desde fuera", por lo tanto se puede ver este texto como base de la comparación de condicio iuris y condicio extrinsecus ${ }^{37}$.

El fragmento de D. 36,2,6,1 $1^{38}$ también habla de extrinsecus en este contexto. La frase "non ex ipso testamento" recuerda a la definición de esta condición en D. $35,1,99$, donde se refiera a ella diciendo "non ex testamento venientes". El jurista Paulus, secundando aquí la opinión de Juliano, constata, que acontecimientos que vienen "desde fuera" no convierten el legado en uno condicional, ya que no

${ }^{36}$ D. 35,1,99 (Papin., 18 quaest.): “Condiciones extrinsecus, non ex testamento venientes, id est quae tacite inesse videantur, non faciunt legata condicionalia".

${ }^{37}$ Castán Pérez-Gómez, "Condicio iuris” y “tacita condicio", cit. (n. 4), pp. 37 y 42.

${ }^{38}$ D. 36,2,6,1 (Paul., 3 Sab.): "At si extrinsecus suspendatur legatum, non ex ipso testamento: licet ante decedat legatarius, ad heredem transmississe legatum dicimus: veluti si rem dotalem maritus legaverit extero et uxori aliquam pro dotali re pecuniam, deinde deliberante uxore de electione dotis decesserit legatarius atque legatum elegerit mulier, ad heredem transire legatum dictum est. idque et Iulianus respondit: magis enim mora quam condicio legato iniecta videtur". 
se considera como condición sino más bien como demora (mora). Especifica como ejemplo el caso de que alguien legue a un tercero un objeto cualquiera de la dote, y a su mujer le prometa una cantidad de dinero junto con la opción del objeto concreto de la dote. El legado del objeto no será condicional, es decir dependiente de la opción de la mujer, ni tendrá efectos en el dies cedens, sino que se considerará como puro. ¿Se puede comparar el caso presente con los ejemplos de D. 36,2,25,1, el hijo que llegará a nacer y los frutos que todavía no existen?

En el fragmento D. $36,2,5,5^{39}$, el jurista Ulpiano constata que aquellas condiciones no tienen efectos por cuanto su verificación depende, no del legatario, sino del heredero o de una tercera persona. Por ejemplo, no produce efectos la condición de que el legatario tenga la obligación de pagarle una cantidad de dinero al heredero, que se niega a aceptarlo. Así mismo se trata el legado como puro si está bajo la "condición" de que el legatario se case con Seya. Estos ejemplos que hacen depender la condición de acciones de terceras personas coinciden con el ejemplo del fragmento D. 36,2,6,1 de la mujer con opción del objeto legado, y también se pueden ver como extrinsecus, es decir circunstancias "desde fuera". Que se trate estos ejemplos como casos de la condicio extrinsecus sin ningun efecto jurídico parece coherente. Pero el fragmento D. 36,2,25,1 resulta problemático, ya que los ejemplos no concuerdan. No se trata de acciones arbitrarias de terceros (a excepción del pupillus, que quizás pagará) sino de circunstancias exteriores en las que no se puede influir, como por ejemplo el nacimiento de un hijo o el crecimiento del fruto. Por qué el jurista ha decidido que a ésas condiciones tampoco se las considere como condiciones propias, no resulta claro. Kaser presenta tres soluciones posibles ${ }^{40}: i$ ) por una parte, Papiniano podría haber considerado todo estos casos como condiciones implícitas (por eso la explicación en el caso del pupillus) las cuales son para él condiciones impropias, porque el hecho de ser implícitas indica que los efectos jurídicos no estaban previstos por la voluntad de las partes; ii) la segunda suposición es que si los ejemplos en cuestión son casos de la condicio iuris, carecen sin embargo de una disposición del Derecho objetivo; iii) la tercera tesis presupone que se trata en todo los ejemplos de condiciones implícitas, que -referente a la interpretación de la voluntad de las partes- provienen "desde fuera", es decir, son "extrinsecus", ya que la interpretación también viene desde fuera.

Está claro que Papiniano se refiere a una condicio tacita en el caso de la "deuda" del pupillus. El texto sólo tiene sentido si se considera los ejemplos del hijo no nacido y de los frutos no crecidos como condiciones tácitas (stricto sensu). La disposición del testador dice expresamente que los frutos en aquel fundo serán legados, pero implícito contiene la condición “si los frutos llegan a existir”. Eso

${ }^{39}$ D. 36,2,5,5 (Ulp., 20 Sab.): "Item si qua condicio sit, quae per legatarium non stat quo minus impleatur, sed aut per heredem aut per eius personam, in cuius persona iussus est parere condicioni, dies legati cedit, quoniam pro impleta habetur: ut puta si iussus sim heredi decem dare et ille accipere nolit. sed et si ita mihi legatum sit, si Seiam uxorem duxero, nec ea velit nubere, dicendum erit diem legati cedere, quod per me non stat, quo minus paream condicioni, sed per alium stat, quo minus impleatur condicio".

${ }^{40}$ Véase: KASER, Symbolae Taubenschlag, cit. (n. 2), p. 430. 
explicaría por qué Papiniano declara en el fragmento siguiente, D. 35,1,99, que la condicio extrinsecus viene "desde fuera" del testamento y que es implícita ("id est quae tacite inesse videantur"). Ése "tacite inesse" se refiere al "quasi tacite condicio inserta sit” del fragmento D. 36,2,25,1, ya que Papiniano identifica la condicio tacita y la condicio extrinsecus.

Como estas condiciones tienen su origen más bien en la interpretación de la voluntad que en el Derecho objetivo (como es el caso de la condicio iuris) -siguiendo la definición de la condicio iuris- se puede poner en duda la calificación de la condicio iuris como condicio extrinsecus. Por consiguiente, la condicio extrinsecus se aplica en dichos casos tanto a la condicio tacita como a los casos de acciones de terceros.

\section{CONCLUSIÓN}

Como se ha puesto claramente de manifiesto en los citados fragmentos, tanto la condicio supervacua como la condicio extrinsecus no tienen efectos jurídicos. En las fuentes sobre la condicio supervacua podríamos sobreentender que el testador sólo quería otorgar un legado y que impuso la condición por error o distracción. En el texto D. 36,2,25,1 al legado se le da otra interpretación conforme con la voluntad del testador, por eso parece coherente suponer que Papiniano negó los efectos de las condiciones (a saber, el aplazamiento del dies cedens) en los casos que él consideraba condicionados por una condicio tacita, por no haber coincidencia con la voluntad del testador.

La única descripción de la condicio iuris en las fuentes se encuentra en el texto D. $35,1,21$, que las califica como un tipo de condiciones "quae vero ex iure venient". La definición y el concepto moderno que conocemos en la actualidad se ha ido formando más bien durante el siglo XIX ${ }^{41}$. Parece que Castán Pérez-Gómez se basa en esta definición, por lo tanto creo que las nociones de la condicio iuris en los textos D. 35,1,21 y D. 21,1,43,10 no coinciden claramente con su definición de la condicio iuris.

Si utilizamos la definición moderna de la condición de Derecho podemos extender su campo de aplicación a las estipulaciones de la dote y a las disposiciones testamentarias, ya que en ellas están incluidos tanto el estado de pendencia como su origen en el "Derecho". En el caso de la aditio hereditatis Castán Pérez-Gómez aplica la condicio iuris, porque la aceptación de la herencia es esencial para que los legados produzcan efectos, lo mismo que en la dote, ya que no hay dote sin subsiguiente matrimonio. Pero en realidad hay que destacar que en las fuentes sobre la estipulación de la dote no existe mención alguna de la condicio iuris pero sí de la condicio tacita.

Tampoco en las fuentes sobre la aditio hereditatis se nombra ni una sola vez la condicio iuris, sólo se habla de la condicio supervacua, que no hay que identificarla

${ }^{41}$ Véase también: Oertmann, Paul, Die Rechtsbedingung (Leipzig, 1924, reimpresión Aalen, Scientia Verlag 1968); Dernburg, Heinrich, Pandekten. Allgemeiner Teil und Sachenrecht (Berlin, 1902), I, p. 246, dice que la condicio iuris es tal condición "welche ein Geschäft von Rechts wegen an künftige ungewisse Ereignisse knüpft”. 
de forma automática con la condicio iuris; esto mismo hay que aplicarlo en el caso de la condicio extrinsecus. El origen de la condicio iuris "ex iure" se encuentra expresado claramente en las fuentes, pero no así el de "extrinsecus", "desde fuera”. Incluso si aplicamos la definición (moderna) de la condición de Derecho a la condicio extrinsecus, sigue habiendo contradicción en el texto D 36.2.25.1, ya que es obvio que los ejemplos que Papiniano da como extrinsecus son casos de la "condicio tacita propiamente dicha" 42 .

Por consiguiente creo que no resulta apropiado aplicar conceptos y definiciones modernas al interpretar las fuentes sobre la condicio iuris en el Derecho romano. Si nos referimos a las fuentes tradicionales sobre la condición de Derecho llegamos a la conclusión de que no es correcto aplicar de forma uniforme el concepto de la condición de Derecho y que en ciertos casos la condicio iuris no se presenta como condición, sino más bien como presupuesto o requisito de validez.

[Recibido el 27 de enero y aprobado el 31 de julio de 2010].

\section{BiBLIOGRAFÍA}

Castán Pérez-Gómez, Santiago, "Condicio iuris" y "tacita condicio". Las condiciones implícitas en el Derecho privado romano (Madrid, Universidad Rey Juan Carlos Dykinson, 2006).

Dernburg, Heinrich, Pandekten. Allgemeiner Teil und Sachenrecht (Berlin (1902), I.

KaSer, Max, "Condicio iuris" und "condicio tacita", en Symbolae Raphaeli Taubenschlag dedicatae (1956), I = El mIsmo, Ausgewählte Schriften (Napoli, Jovene, 1976), II.

KöNIG, Hans-Herbert, Die vor der Ehe bestellte dos nach klassischem römischen Recht, en SDHI., 29 (1963).

LÉGIER, H.-J., “Tacita condicio”, en RHDFE., 44 (1966).

Masi, Antonio, Studi sulla condizione nel Diritto romano (Milano, Giuffrè, 1966).

Oertmann, Paul, Die Rechtsbedingung (Leipzig, 1924, reimpresión Aalen, Scientia Verlag, 1968).

Riccobono, Salvatore, Stipulatio ed instrumentum nel Diritto giustinianeo, en ZSS., rom. Abt., 43 (1922).

WACKE, Andreas, Zur causa der Stipulation, en TR., 40 (1972).

Wolf, Joseph Georg, Causa stipulationis (Köln - Wien, 1970).

\footnotetext{
${ }^{42}$ Por una parte, el texto habla expresamente de la condicio tacita. Además los ejemplos coinciden con la definición de la "condicio tacita propiamente dicha" que da Castán PérezGómez.
} 